\title{
A NEW APPROACH FOR SPECTRORADIOMETRIC CALIBRATION CONSISTENCY ON THE GROUND AND IN SPACE
}

\author{
Donald F. Heath ${ }^{\mathrm{a}}$ and Georgi Georgiev ${ }^{\mathrm{b}}$ \\ ${ }^{a}$ Heath Earth/Space Spectroradiometric Calibration Consulting, LLC., 2883 Springdale Lane, Boulder, CO 80303 \\ ${ }^{\mathrm{b}}$ Sigma Space Corp., 4600 Forbes Blvd., Lanham, MD 20706
}

\begin{abstract}
A Space-based Calibration Transfer Spectroradiometer (SCATS) is combined with a ground calibration spectral albedo radiometric standard which consists of an opaque quartz glass Mie scattering diffuser (MSD) which has very good Lambertian scattering properties in both reflectance and transmittance modes. This system provides the capability for determining long term changes in the spectral albedo calibrations which operate in the solar reflective wavelength region. The spectral albedo calibration would be traceable to the SIRCUS and STARR NIST calibration facilities. The on-orbit radiometric standard is the Sun. The NIST traceable ground spectral albedo calibration is invariant between the ground and on-orbit over the instrument lifetime due to the use of a field of view defining mechanical baffle to differentiate between radiance and irradiance.
\end{abstract}

Key words: Lambertian volume diffuser, Mie scattering, radiometric calibration, inter-calibration of space instruments, NIST

\section{INTRODUCTION}

In the solar reflective wavelength region the spectral albedo calibrations of space instruments are tied often to either the spectral albedo of a solar diffuser or the Moon. The Space-based Calibration Transfer Spectroradiometer (SCATS) sensor uses a simple, invariant optical configuration and dedicated narrow band spectral channel modules to provide very accurate, polarization-insensitive, stable spectral measurements of earth albedo and lunar disk albedo. Optical degradation effects on calibration stability are eliminated through use of a common optical system for observations of the Sun, Earth, and Moon. The measurements from space would be traceable to SI units through preflight calibrations of radiance and irradiance at NIST's SIRCUS facility and the invariant optical system used in the sensor. Simultaneous measurements are made in multiple spectral channels covering the solar reflective wavelength range of $300 \mathrm{~nm}$ to 2.4 microns. A mini spectrograph can be used to provide interpolation of the spectral albedo channels. The large dynamic range of signals is handled by use of photodiodes which are highly-linear detectors, stable discrete electronic components, and a non-imaging optical configuration. By observing the Sun on every orbit, the most stringent stability requirements of the system are limited to short time periods. The invariant optical system for both radiance and irradiance measurements also give excellent transfer to-orbit SI traceability. A Mie scattering diffuser (MSD) which is fabricated from opaque quartz glass from Heraeus Quarzglas is used to transfer a NIST derived spectral albedo calibration to SCATS, and other space instruments in the solar reflective wavelength region. During the course of characterizing MSDs in the thermal infrared a new type of MSD was discovered which is based on restrahlen radiation from both synthetic and natural forms of opaque quartz glass in the $5-25 \mu \mathrm{m}$ wavelength region. The orbital views of SCATS, signal ranges and observational time lines are shown in Fig.1.

\section{SCATS CONCEPTUAL DESIGN AND MEASUREMENT CONCEPT}

The basic SCATS instrument is designed to accommodate 19 photometer channels in a one axis rotating scan head scanning perpendicular to the orbital plane. Each photometer channel consists of a mechanical baffle, a narrow band optical filter, and a detector. The $\mathrm{f} / 8$ mechanical baffle determines the IFOV when observing Earth radiance. The baffle design has been shown to provide an out-of-field rejection $>10^{4}$. Precision apertures are used in 
front of the photodiode windows to adjust the signal range. Nominally, 10 silicon photodiodes are used in combination with high stability ion-assisted-deposition narrow band interference filters (FWHM $\sim 10 \mathrm{~nm}$ ) to cover the wavelength range from $300 \mathrm{~nm}$ to $1,000 \mathrm{~nm}$. A nominal 9 cooled InGaAs photodiodes are used with a series of ion-assisted-deposition (IAD) filters (FWHM $\sim 0.1 \mu \mathrm{m}$ ) for measurements in the 1000 to $2400 \mathrm{~nm}$ wavelength region. The detectors that are proposed for use have been shown to exhibit a high degree of linearity over more than six orders of magnitude by national measurement laboratories (NML). e.g. Yoon et al., 2003 [1]; Durak and Aslan, 2004 [2]. Measuring detector linearity is based on the beam conjoiner technique (Saunders and Shumaker, 1984 [3]; Thompson and Chen, 1994 [4].
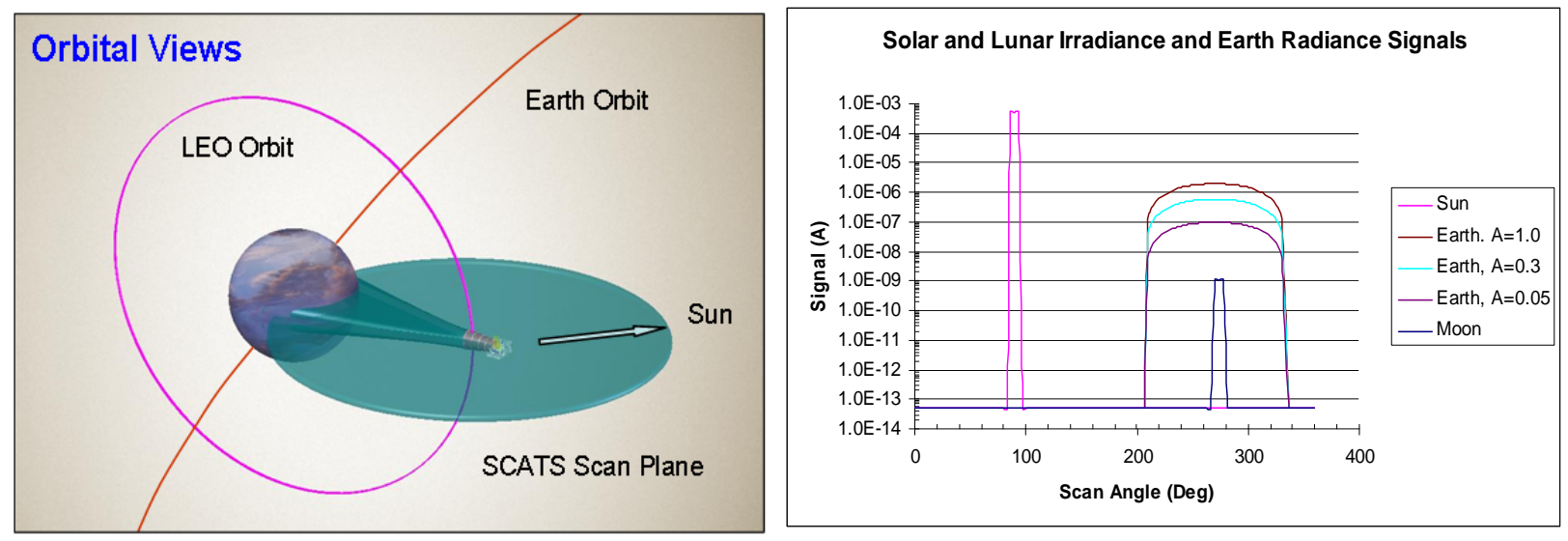

Fig. 1. The SCATS scan is perpendicular to the orbital plane. Solar and lunar measurements are made using the orbital motion to scan through the Sun or the Moon. The figure on the right shows an example of calculated signals for solar, lunar (full phase), and earth observations for albedos of 1.0, 0.3, and 0.05. The scan head uses a single axis of rotation to view the Sun, Earth, or Moon. A typical orbital period is $\sim 100$ minutes.
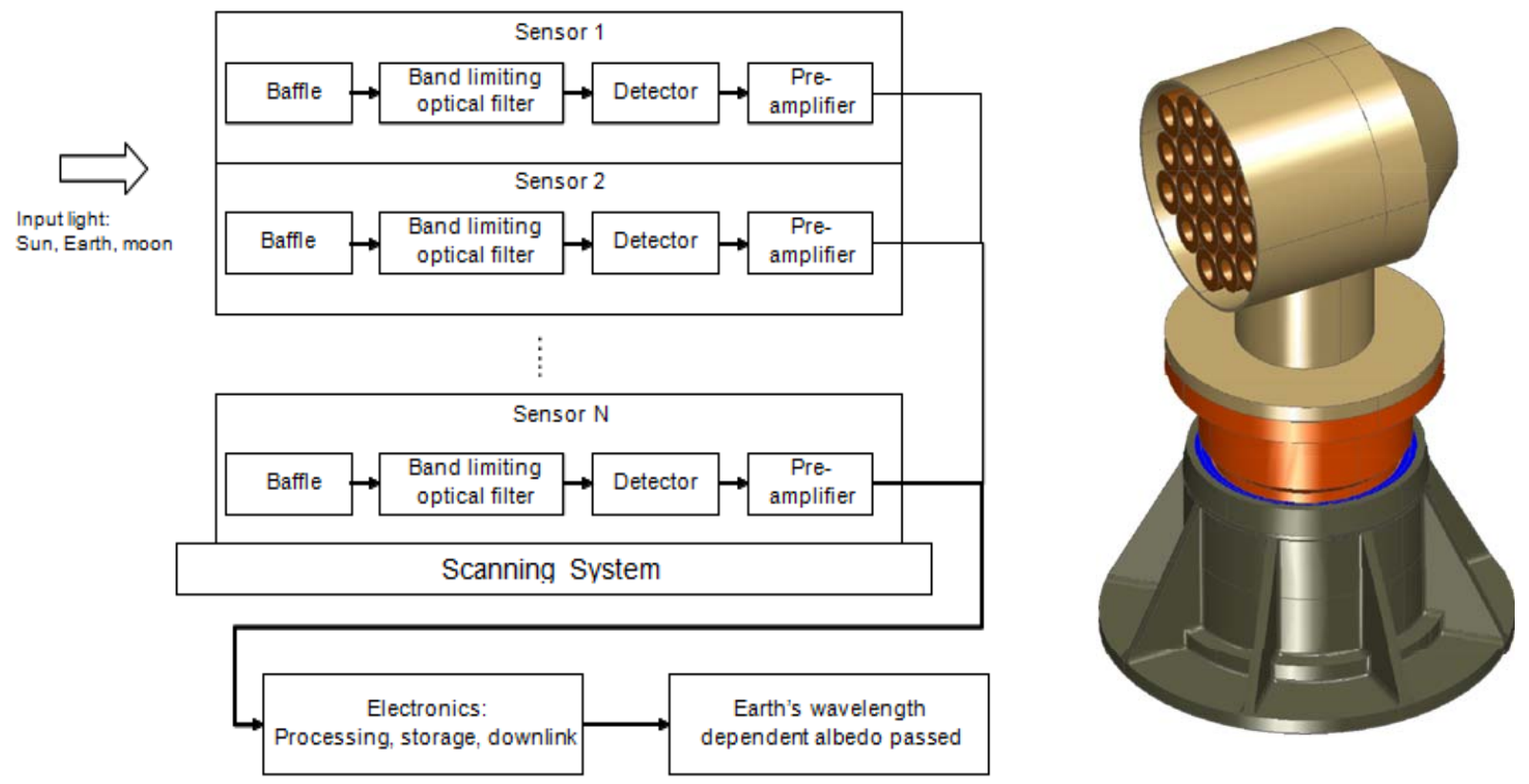

Fig. 2. A block diagram and a 19-channel SCATS conceptual model are shown. The co-aligned sensors make simultaneous observations of the solar irradiance, the lunar irradiance, or the terrestrial nadir or horizontal scan radiances using a single rotational axis scan head. 
Critical elements in the SCATS instrument are the short and long-term radiometric stability of the optical filters. The narrow band interference filters are fabricated using the IAD or some other comparable technique that produces hard filter coatings with no voids for the absorption of water vapor. New filter materials such as the rare earth oxide coatings are being used to fabricate filters with low thermal coefficients. The use in space requires that the effects of a high vacuum environment, ultraviolet solar radiation, high energy charged particles, changes in the thermal environment due to solar and spacecraft heating, the F-number of the baffle, and ageing should have a negligible effect on the filter central wavelength and spectral shape. The excellent performance of this new class of hardened filters maintains SCATS spectral response stability and has been discussed extensively in the literature by Takashashi (1995), Martin et al. (1983), and Allan et al. (1999). A detailed uncertainty analysis has been performed which is based on Type A and B uncertainties. The results are contained in a Ball Aerospace \& Technologies Corp. System Engineering Report by Tarde and Heath (2009) [5]. The top level uncertainty breakdown is shown in Fig. 12. This report is available upon request. In Table 1, the uncertainty of the simultaneous nadir overpass technique is probably the least well known component in the uncertainty budget. A description of recent ongoing uses of this technique is given by Cao et al., 2007 [6].

Table 1. SCATS uncertainty budget

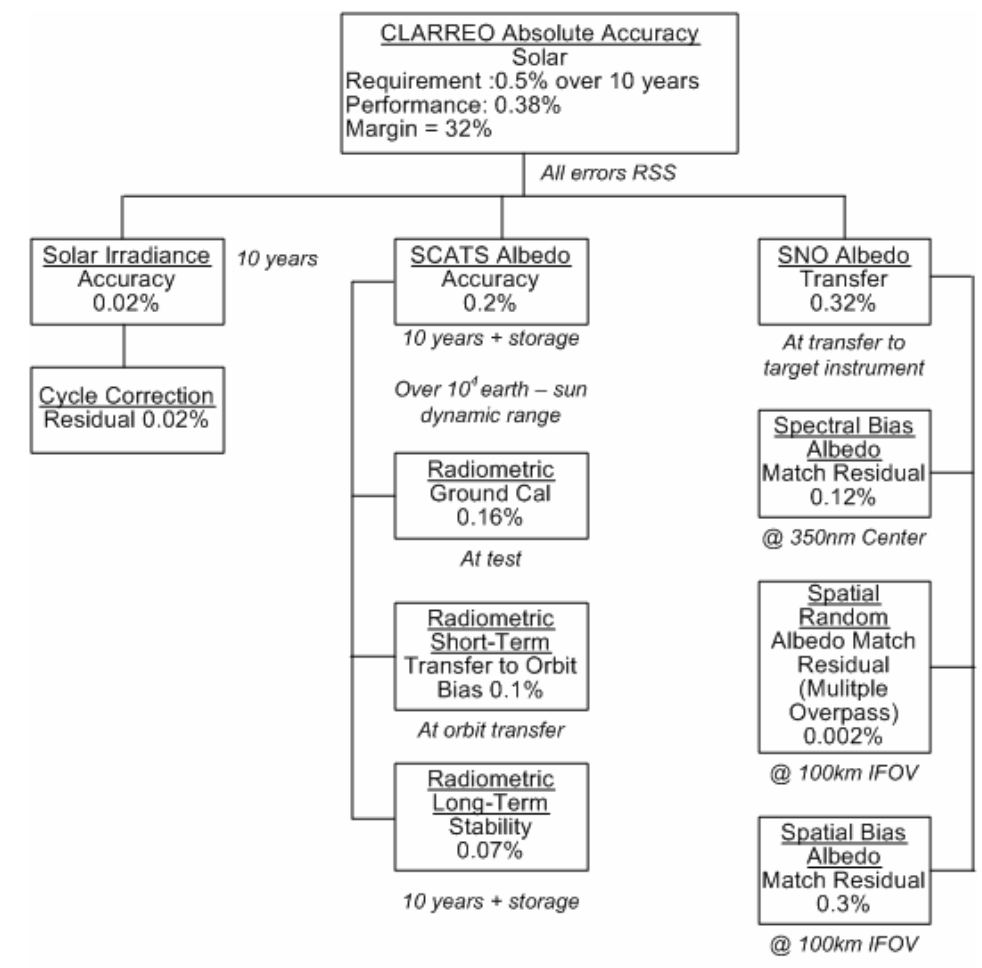

\section{CHARACTERIZATION OF MIE SCATTERING DIFFUSERS}

The concept for the use of a Mie Scattering Diffusers (MSD) as a SI traceable spectral albedo radiometric standard was developed by D. F. Heath. Its SI units traceability is derived from measurements of its bidirectional reflectance distribution function (BRDF) and bidirectional transmittance distribution function (BTDF) by the NIST Spectral Tri-function Automated Reference Reflectometer (STARR) facility which has a measurement uncertainty of $0.5 \%(\mathrm{k}=2)$ for the wavelength range from $200 \mathrm{~nm}$ to $2.5 \mu \mathrm{m}$. A description of the extension of the NIST BRDF scale from $1100 \mathrm{~nm}$ to $2500 \mathrm{~nm}$ is given by Yoon et al., 2009 [1]. The new paradigm for SI traceable radiometric calibration as advocated by Pollock et al., 2003 [7] and Datla et al., 2009 [8] and 2010 [9]. The MSD approach has used primarily a fused silica material fabricated by Heraeus Quarzglas known as OM-100. This material is fabricated from fused silica which contains irregular shaped voids which are of the order of $20 \mu \mathrm{m}$ across and make up approximately $2.3 \%$ of the volume of fused silica. This paper describes preliminary results from characterization measurements of a $2 \times 2$ " x $3.0 \mathrm{~mm}$ thick sample and a $12 \times 12$ " x $10.0 \mathrm{~mm}$ thick samples of 
Heraeus OM-100 that was fabricated from natural fused silica, pegmatite. There are approximately $10^{\wedge} 5-10^{\wedge} 6$ voids $/ \mathrm{cm}^{\wedge} 2$ in the $3 \mathrm{~mm}$ thick sample. These voids are randomly distributed in order to avoid coherence effects which result in spurious fine structure features. Results described in a subsequent section indicated the need for a synthetic form of a MSD. Heraeus Quarzglas fabricated a puck from synthetic fused silica. Both front and rear surfaces were optically ground with 180 grit (average grit size $76 \mu \mathrm{m}$ ), etched, and cleaned prior to the measurements described in this paper and previous papers by Heath and Georgiev [10]. The principal purpose was to remove the specular reflective component.

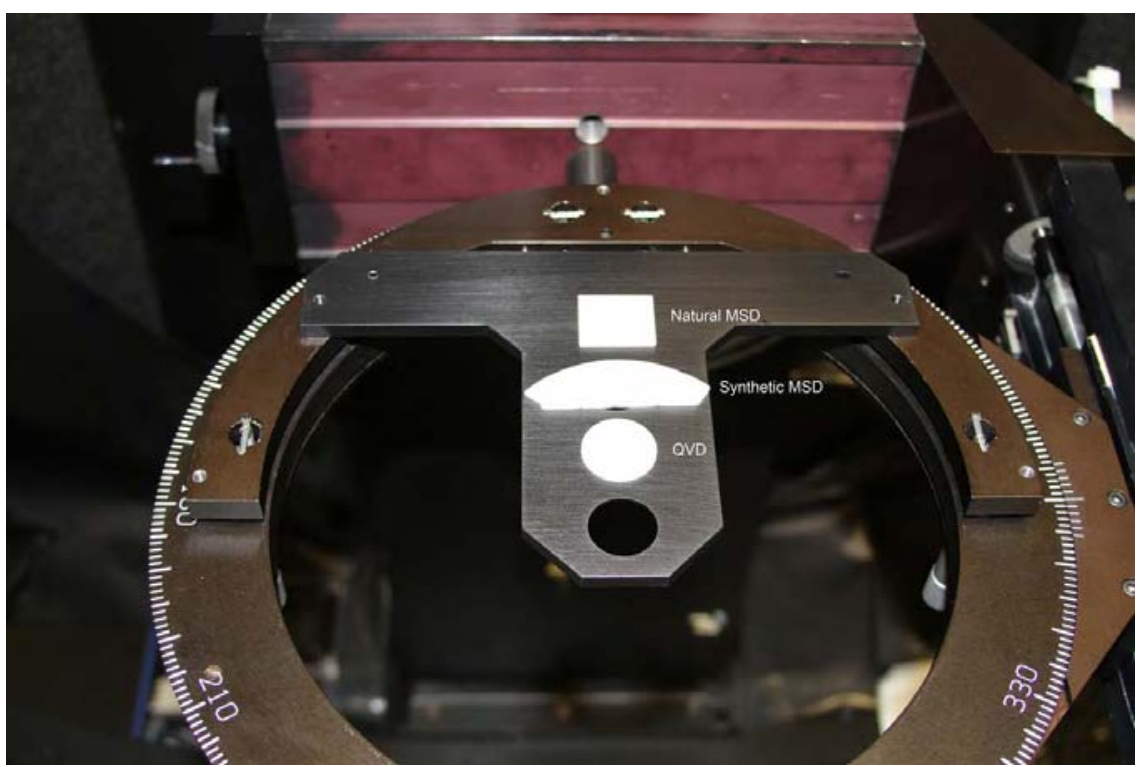

Fig. 3. A partial view of the GSFC measurement system with the natural and synthetic OM 100 MSDs and a QVD of the type flown on the OMI instrument.

Mie Scattering Diffuser at $325 \mathrm{~nm}, \mathrm{AOI}=0^{\circ}$

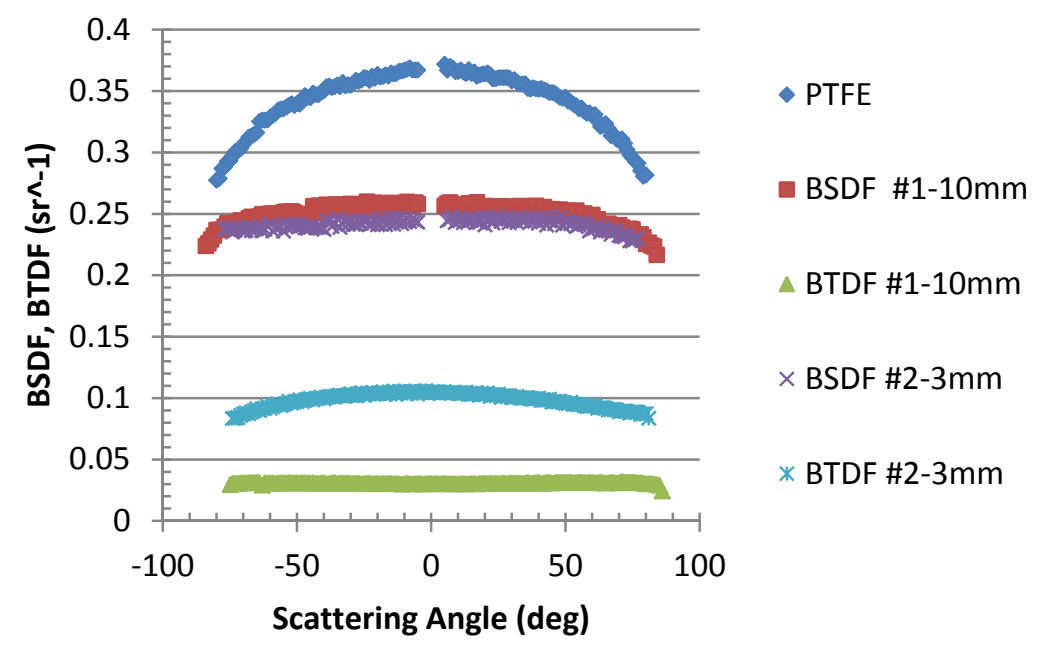

Fig. 4. A comparison is shown between the BSDF of PTFE a.k.a. Spectralon ${ }^{\mathrm{TM}}$ and 3 and $10 \mathrm{~mm}$ thick MSDs. Also shown are the corresponding BTDF values for the MSDs. This figure also illustrates the higher Lambertian scattering properties of the MSDs over PTFE.

A quasi-volume diffuser (QVD) was designed by TPD at the Technical University of Delft. It is fabricated from UV grade synthetic fused silica. Both front and rear surfaces are optically ground and the back ground surface 
is coated with vacuum deposited aluminum which is designed to convert a solar irradiance into a radiance source. The thickness of the fused silica disc is about $3 \mathrm{~mm}$. The purpose of the QVD is to transform an irradiance source into a radiance source and to destroy spatial coherency effects which were a major source of noise in the OMI solar irradiance measurements.

Mie scattering diffusers which are calibrated for BSDF and BTDF at the NASA's GSFC facility can be used in either a reflection or transmission mode. Theoretical studies on the physical properties of MSDs indicate that the greatest accuracy is most likely to be realized when working in the transmission mode. Reasons for this are that spatial coherency effects are expected to be less, incident polarized radiation is completely depolarized, there is no specular component (Bicout, et al., 1994) [11], and optical aberrations are likely to be less in an on axis illumination with a radiometric standard source. The preliminary results from the characterization of natural and synthetic forms of MSDs have been described by Heath and Georgiev (2011) [10].

It is important to note that the thickness of the synthetic MSD is $2.93 \mathrm{~mm}$, the natural MSD is $2.04 \mathrm{~mm}$, and the thickness of the QVD is $2.04 \mathrm{~mm}$. The different thicknesses for the synthetic and natural MSDs will increase the BRDF of the synthetic MSD and decrease the BTDF. These differences in the thicknesses will show up in measurements of the diffuse reflectance and transmittance, BRDF and BTDF. The MSD measurements of BRDF and BTDF are shown in Figures 5 and 6. Corresponding measurements of BRDF are shown in Figure 7 for the QVD which exhibits a negligible thickness effect. The tabulated values for these figures are placed at the end of this paper in Appendix I.

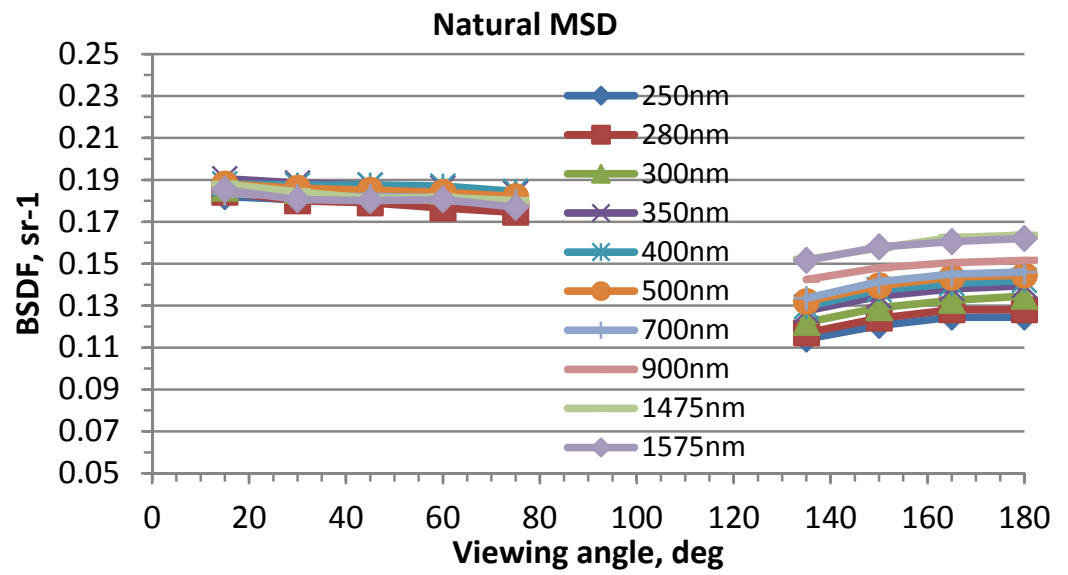

Fig. 5. Graph of data in Table 2 for BRDF and BTDF of natural OM-100 opaque quartz glass.

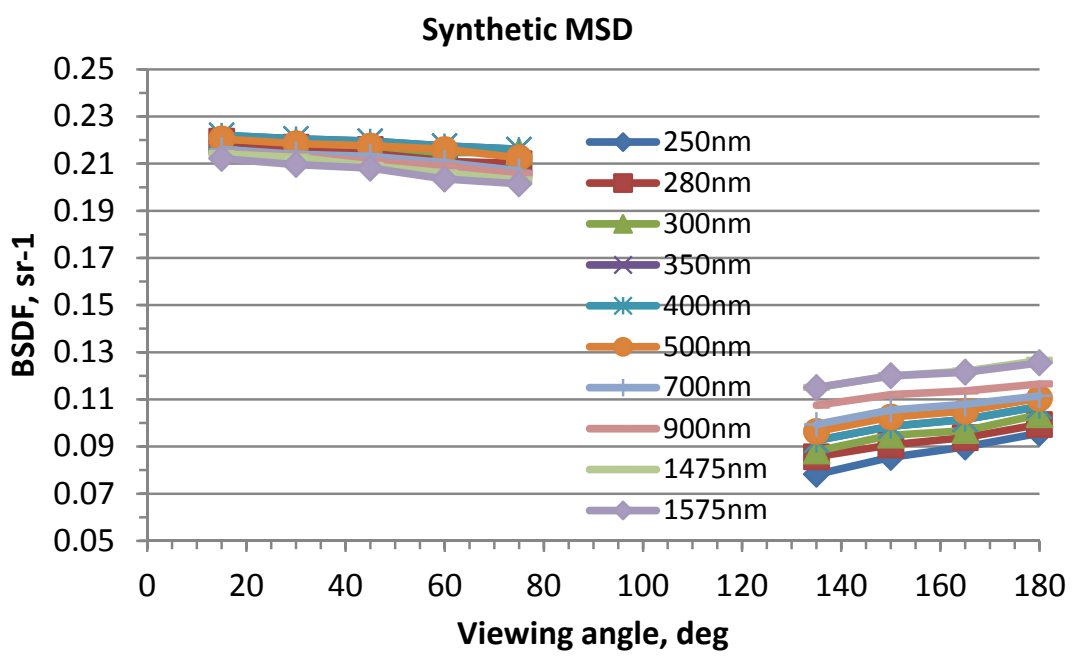

Fig. 6. Graph of data in Table 3 for BRDF and BTDF of natural OM-100 opaque quartz glass. 


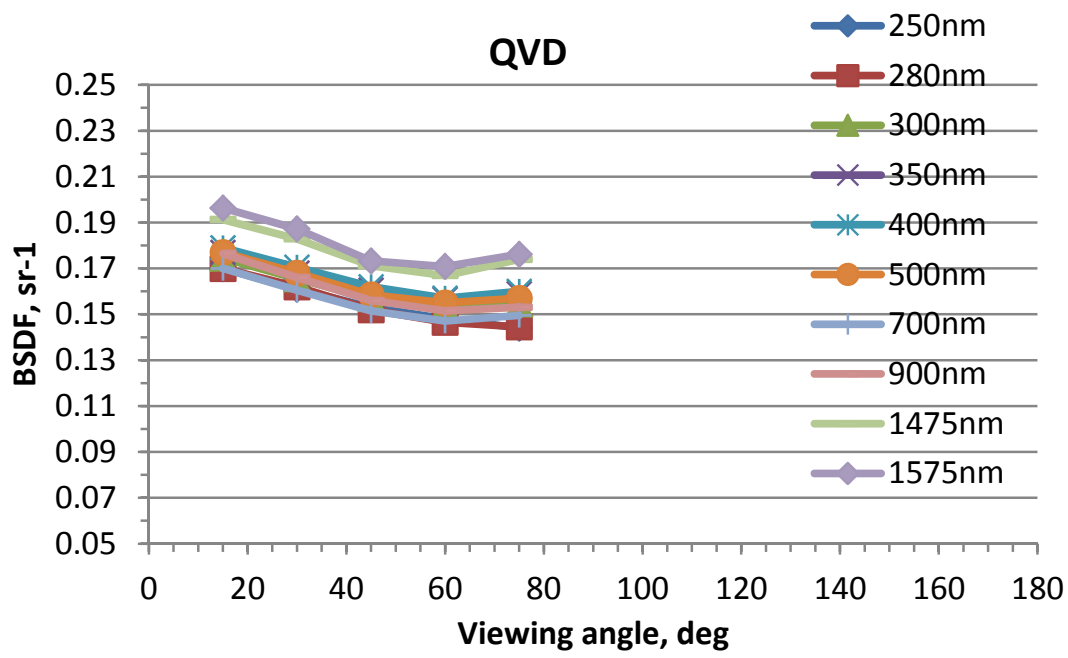

Fig. 7. Graph of data in Table 4 for OMI type QVD.

The shapes of the distributions of BRDF and BTDF for the synthetic and natural forms of the MSDs are similar. The principal differences are in the magnitude of the values which are due principally to the difference in the thickness. A comparison of the BRDFs between the synthetic MSD and the QVD indicate that the MSD is more Lambertian and show smaller wavelength dependence as a function of scattering angle. This is particularly noticeable for 1475 and $1575 \mathrm{~nm}$.

\section{SPACE ENVIRONMENT EFFECTS ON MSDS}

The transmittance sensitivity of synthetic fused silica MSDs to some of the effects of a space environment were investigated. The dimensions of the samples are $24 \times 24 \times 2.0 \mathrm{~mm}$ for the natural MSD and $24 \times 27 \times 2.0 \mathrm{~mm}$ for the synthetic MSD. It became clear from earlier measurements of the effects of $\mathrm{Co}^{60}$ gamma rays that natural fused silica impurities were producing a significant absorption by color centers in the ultraviolet. Gamma ray absorption effects on irradiated and heat-treated natural quartz effects are discussed in the paper by Nunes and Lameiras, 2005 [12].

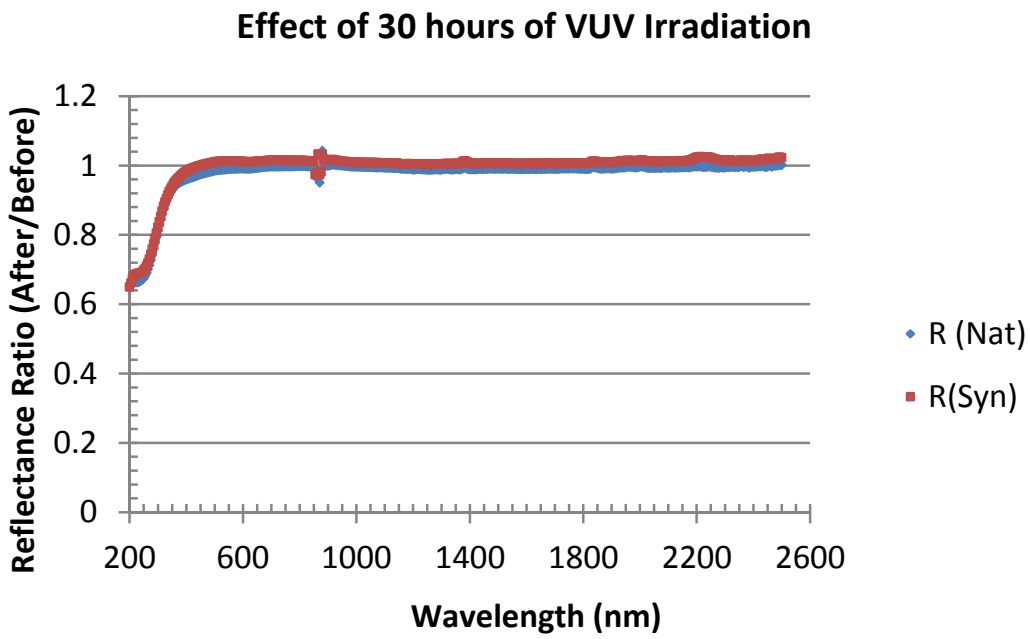

Fig 8. Effect of $\sim 7$ equivalent Sun hours of irradiation in the $115 \mathrm{~nm}$ to $165 \mathrm{~nm}$ region from a McPherson 632 vacuum ultraviolet deuterium light source equipped with a magnesium fluoride window on the diffuse reflectance of natural and synthetic MSDs. 
The dimensions of the samples are $24 \times 24 \times 2.0 \mathrm{~mm}$ for the natural MSD and $24 \times 27 \times 2.0 \mathrm{~mm}$ for the synthetic MSD. These are the samples which were irradiated with VUV radiation from a deuterium lamp down to $115 \mathrm{~nm}$ and which overlap the wavelength region of the ambient xenon arc. The results of these measurements are given in Figure 7. The major effect appears to be the reduction in transmission in the short wavelength cutoff region. This decrease in transmittance is most likely the formation of color centers associated with impurity atoms. A more detailed discussion of these effects is contained in the paper by Heath and Georgiev (2011) [10].

\section{HEMISPHERICAL REFLECTANCE AND TRANSMITTANCE from $205 \mathrm{~nm}$ to $25 \mu \mathrm{M}$}

A graphical representation is shown Figures 8 and 10 of the diffuse reflectance and transmittance measurements for the 3-mm thick synthetic and for the 2-mm thick natural MSD samples for the wavelength region from $205 \mathrm{~nm}$ to $5.0 \mu \mathrm{m}$. The spectral differences between synthetic and natural MSDs are due primarily to the 0.9$\mathrm{mm}$ difference in thickness. The short wavelength cutoff in transmittance is due to absorption by impurity elements. The absorption features at 2.2 and $2.72 \mu \mathrm{m}$ are due to $\mathrm{OH}$. The MSDs become opaque at $5.0 \mu \mathrm{m}$. A comprehensive study of BRDF of several surfaces as a mid-wave infrared diffuse reflectance standard has been published recently as Master's Thesis by Balling (2007) [13].

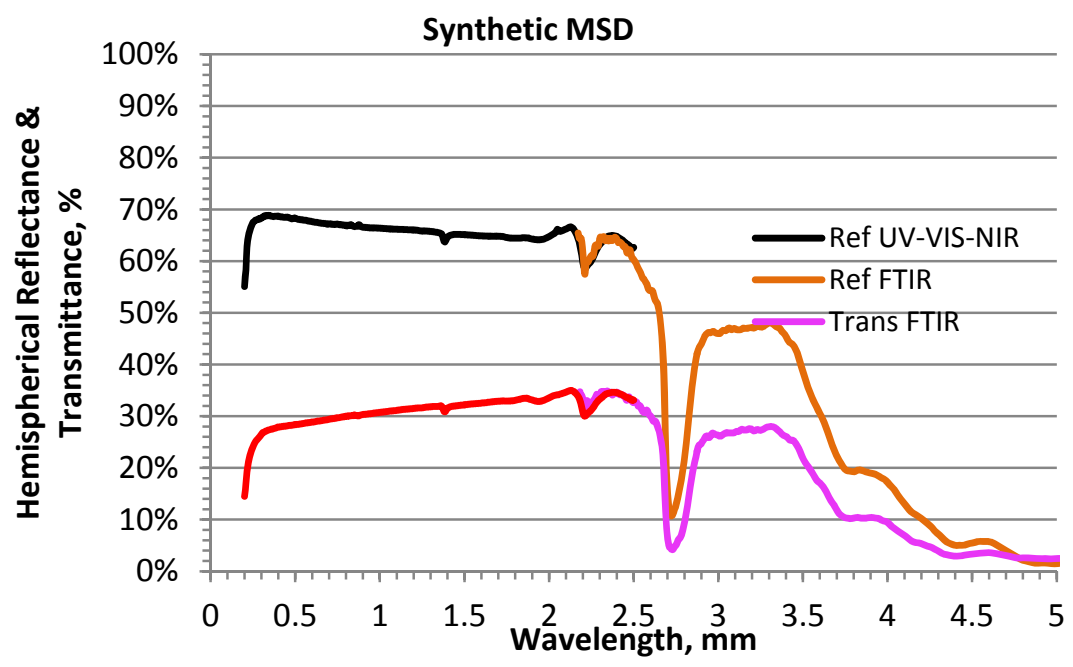

Fig 9. Diffuse reflectance and transmittance of 3-mm thick synthetic MSD from $200 \mathrm{~nm}$ to $5.0 \mu \mathrm{m}$

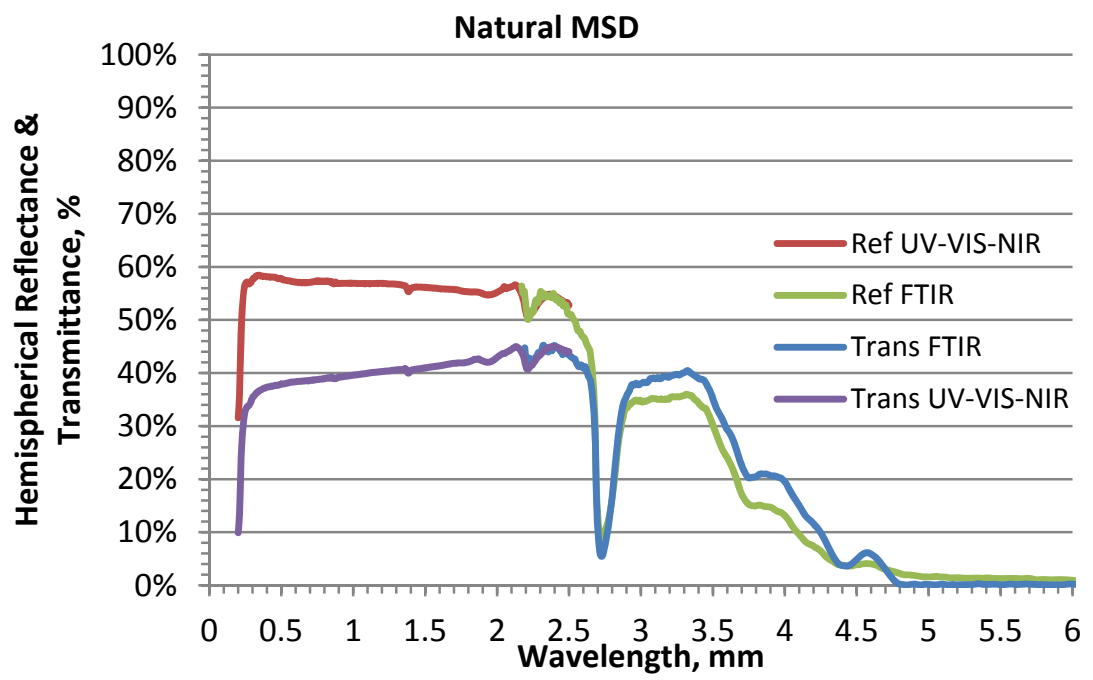

Fig 10. Diffuse reflectance and transmittance of 2-mm thick natural MSD from $205 \mathrm{~nm}$ to $5.0 \mu \mathrm{m}$ 
The hemispheric reflectance and transmittance measurements for the natural and synthetic opaque quartz glass MSDs are very similar in their spectral behavior. The principal differences are due to the $0.9 \mathrm{~mm}$ difference in thickness.

MSD Reflectance + Transmittance

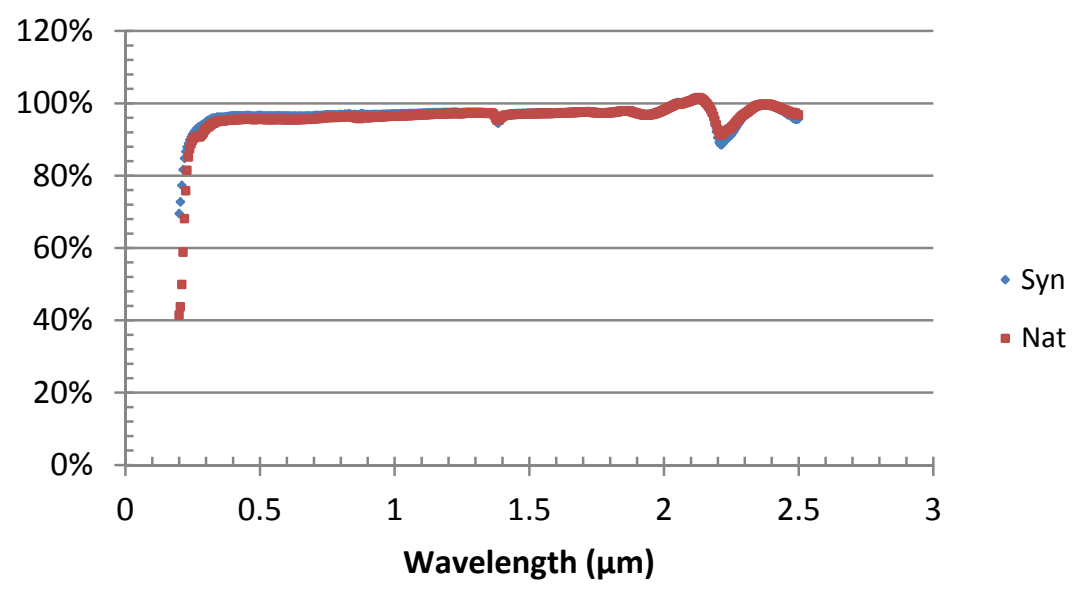

Fig. 11. The sum of diffuse reflectance and transmittance for synthetic and natural MSDs. The weak absorption band $\sim 2.25 \mu \mathrm{m}$ is due to $\mathrm{OH}$, and the increasing absorption for wavelengths less tha $300 \mathrm{~nm}$ is due to impurities.

An effective way to visualize the presence of absorbing species is to sum the hemispherical reflectance and transmittance. This is shown in figure 10. There is negligible absorption in the spectral region extending from the short wavelength cut-off to $2.5 \mu \mathrm{m}$ except for the small $\mathrm{OH}$ absorption feature. The hemispheric reflectance and transmittance measurements of the synthetic and natural MSDs in the 5-25 $\mu \mathrm{m}$ wavelength region showed surprising behavior in a region which is reported as totally absorbing Kitamura et al. (2007) [14]. There are two wavelength regions of surprisingly high reflectance at 8.9 and $20.9 \mu \mathrm{m}$ which are interpreted as restrahlen radiation from resonance radiation vibrational modes from the Si-O-Si molecule. An early description of restrahlen radiation is by Rubens and Nichols (1897) [15]. A description of bulk and surface reflectance has been given by Suits in Chapter 3 of the Infrared Handbook, revised edition (1989) in which it is stated that restrahlen radiation is primarily a surface phenomenon and that bulk reflectance is absorbed just below the surface. The hemispheric reflectance measurements for the synthetic and natural opaque quartz glass appear to contradict this statement. The $3.0 \mathrm{~mm}$ thick synthetic MSD has a peak reflectance at $8.9 \mu \mathrm{m}$ of $39.0 \%$ whereas the $2.0 \mathrm{~mm}$ natural MSD has a corresponding peak reflectance of $32.5 \%$. Measurements of hemispheric reflectance on a $3.0 \mathrm{~mm}$ thick sample of the natural MSD (Heraeus OM-100) with a polished surface give a peak reflectance of $\sim 53 \%$ at $8.9 \mu \mathrm{m}$. (Dr. Andreas Götzendorfer, private communication, 2012). It is suggested that the differences in the peak reflectance may be related to the difference in thickness of the two MSDs, and hence a volume scattering contribution. The difference between the polished surface of the Heraeus sample and those used for the measurements reported here suggest the possibility of a Lambertian scatterer from the ground surface ( $76 \mu \mathrm{m}$ average diameter grit) and the 20 $\mu \mathrm{m}$ irregular shaped volume voids.

It is suggested that restrahlen MSDs fabricated from Heraeus OM-100 could be used as uniform scattering reflection diffusers at $8.9 \mu \mathrm{m}$ in the thermal infrared. This opens up the possibility of a solar reflection diffuser for calibration of remote sensing instruments in space from $250 \mathrm{~nm}$ to $8.9 \mu \mathrm{m}$ and maybe at $20.9 \mu \mathrm{m}$.

The BRDF measurements for the synthetic and Natural MSDs are given on an expanded scale Figure 13 to show the variation with wavelength. These values are plotted in Figure 14 along with the peak hemispheric reflectances / $\pi$ at 8,9 and $20.9 \mu \mathrm{m}$ to show the relationship to the BRDF measurements in Figure 13 if the thermal infrared measurements were exhibiting Lambertian scattering characteristics. 


\section{Synthetic Quartz Restrahlen MSD}

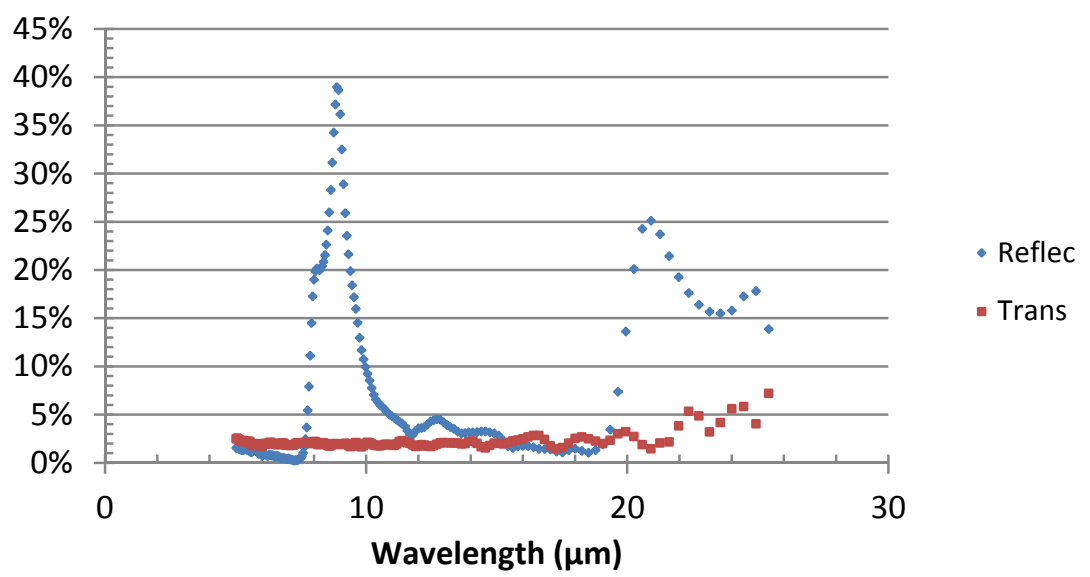

Fig. 12. Measurements of diffuse reflectance and transmittance from 5 to $25 \mu \mathrm{m}$ show significant peaks in reflectance in a wavelength region of strong absorption by quartz glass. These peaks in reflectance at 8.9, 13.1 and $20.9 \mu \mathrm{m}$ are attributed to restrahlen radiation from vibrational resonances of the $\mathrm{Si}-\mathrm{O}-\mathrm{Si}$ asymmetric stretching, the symmetric vibrational stretching of the $\mathrm{Si}-\mathrm{O}-\mathrm{Si}$ bridge, and the O-Si-O bending or to the "rocking" mode of Si-O-Si bonds caused by the displacement of an O atom out of the Si-O-Si plane. Kitamura et al. (2007) have described very strong absorption features in silica glass at $\sim 9-9.5,12.5$, and 21-23 $\mu \mathrm{m}$ due to Si-O-O resonance modes of vibration.

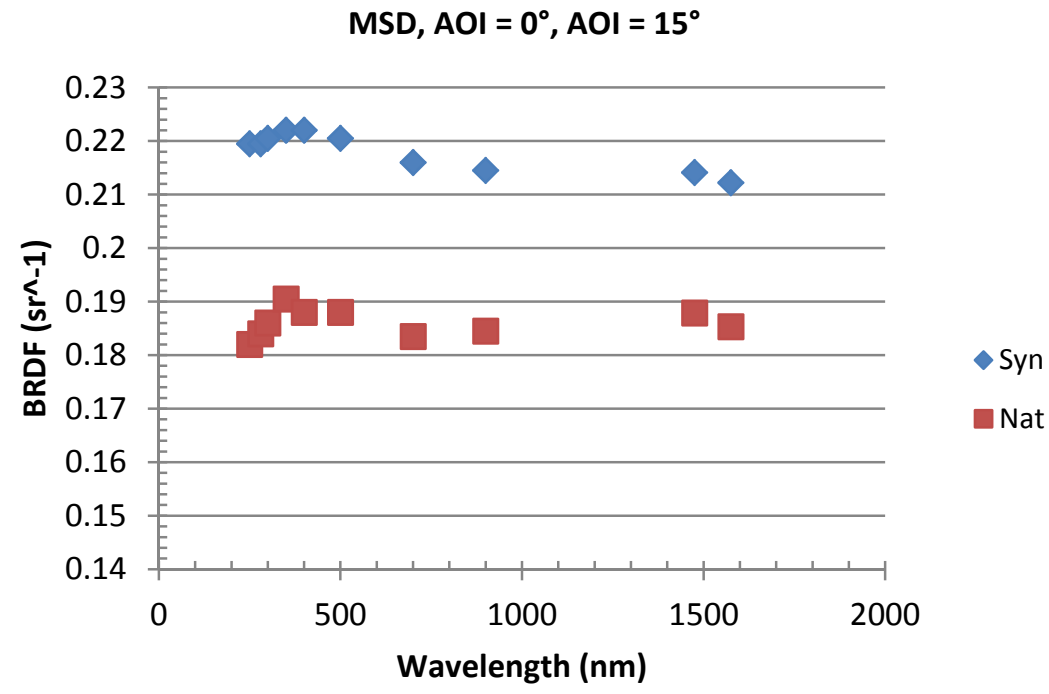

Fig. 13. BRDF for synthetic and natural MSDs for an angle of incidence of $0^{\circ}$ and an angle of scattering of $15^{\circ}$ 


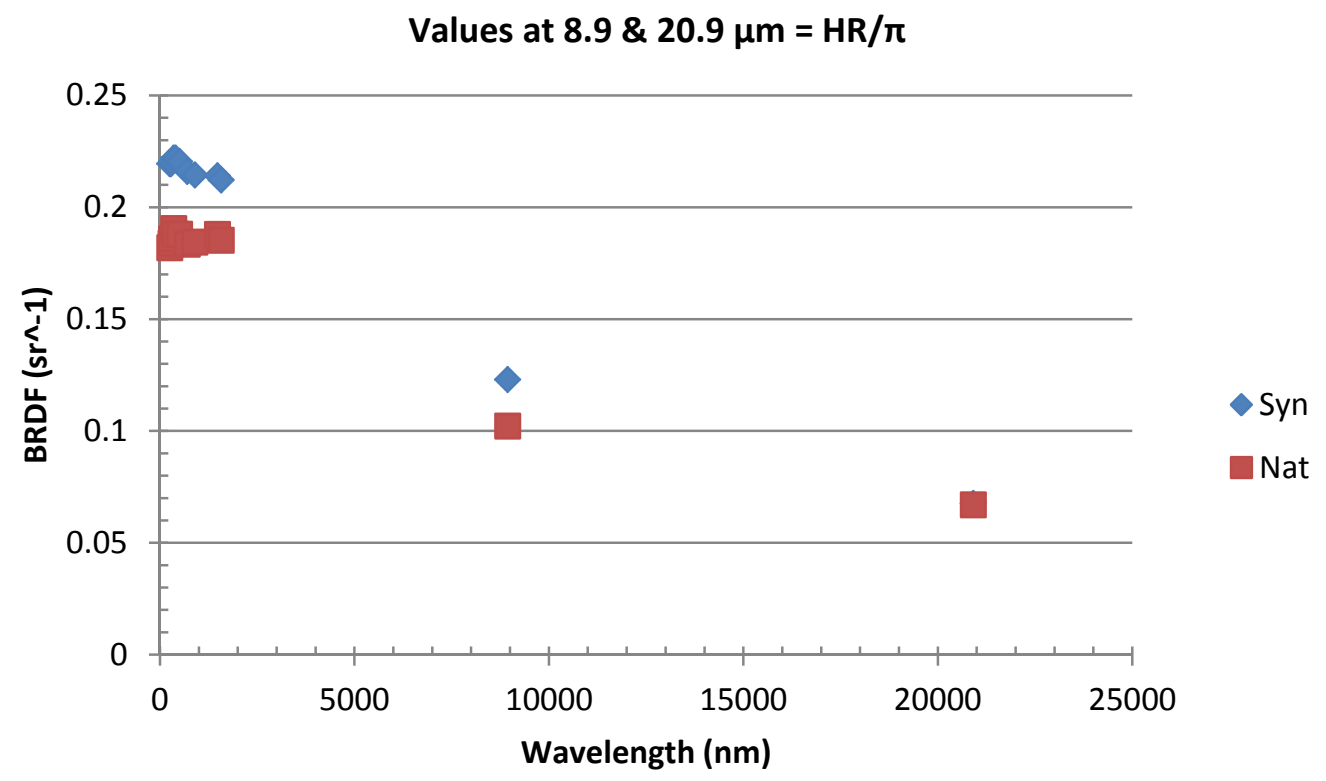

Fig. 14. Comparison of the BRDF values in Figure 13 with the peak hemispheric reflectances at 8.9 and $20.9 \mu / \pi$.

\section{SUMMARY AND CONCLUSIONS}

1. Experimental measurements and analyses have shown that SCATS should be capable of providing a new high level of accuracy for space measurements in the solar reflective wavelength region from $300 \mathrm{~nm}$ to 3.5 $\mu$ mover a decade on-orbit which are traceable to a NIST spectral albedo calibration using the SIRCUS Facility at the $<1.0 \%$ level.

2. This high level of accuracy is achieved through the use of a common optical system which can provide accurate spectral albedo measurements from earth measured earth radiances relative to measured solar and lunar irradiances. The earth radiances are derived using a field defining mechanical baffle. For a typical LEO the earth IFOV of F/8 corresponds to about $100 \mathrm{~km}$. The use of a mechanical baffle in front of the irradiance mode optic to define the radiance mode provides an invariant system which spans the NIST calibration through launch until the end of the instrument lifetime.

3. The SCATS instrument scan head requires only one degree of rotation. A solar or lunar scan is realized by placing the scan head in a fixed position, and letting the orbital motion scan through the Sun or Moon through the optical axis of the system. The system is designed to have no polarization sensitivity. No apertures or any other technology is required to attenuate the solar signal. Linearity measurements at NIST have shown $\mathrm{Si}$ and InGaAs photodiodes can meet the dynamic range and linearity requirements.

4. A mini-spectrograph equipped with a fiber optic input could be positioned in place of one the channels to provide spectral measurements for interpolation of the lunar irradiance and earth radiance signals between the central wavelengths of the co-linear array of photodiode channels. An opaque quartz glass MSD could be placed in in the transmission mode could be placed in the vicinity of the entrance slit in order to depolarize the input radiation.

5. Measurements of the properties of opaque quartz glass Mie scattering diffusers from Heraeus have shown that they exhibit many optical and physical properties which are superior to PTFE, aka Spectralon. ${ }^{\mathrm{TM}}$ These include Lambertian scattering in reflection and transmission, thermal stability at high temperature, extremely low thermal expansion coefficients, a closed pore structure, insensitivity to polar and non-polar solvents, etc. 
6. MSDs can be used as spectral albedo standards from measurements made with the NIST SIRCUS Facility. The intrinsic stability of opaque quartz glass MSDs make them ideal spectral albedo standards for the solar reflective wavelength region from $200 \mathrm{~nm}$ to $3.5 \mu \mathrm{m}$. Since the micro pores make up about $1.0 \%$ of the quartz glass by volume the physical and optical characteristics are similar to clear quartz glass. MSD spectral albedo standards traceable to NIST can be used to transfer spectroradiometric scales to other remote sensing instruments.

7. Characterization of a restrahlen MSD suggest that it could be used potentially as a solar or laboratory calibration diffuser in the thermal infrared with Lambertian scattering properties.

\section{ACKNOWLEDGEMENTS}

We would like to thank Manuel Quijada of NASA's GSFC Optics Branch for his help with the infrared FTIR measurements of diffuse reflectance transmittance of the MSDs at GSFC, and Rich Tarde, Jim Leitch, Jim DeCino at Ball Aerospace for their help in the development of the SCATS instrument concept. We would also like to acknowledge the support of Ashur Atanos of Heraeus Quartz America, LLC., and Dean Spieth of Ball Aerospace and Scott Janz of GSFC for their encouragement and support in the development of the MSD technology.

\section{REFERENCES}

[1] Yoon, H.W., D.W. Allen, G.P. Eppeldauer, and B.K. Tsai, "The Extension of the NIST BRDF Scale from $1100 \mathrm{~nm}$ to $2500 \mathrm{~nm}$," Proc. of SPIE Vol. 7452, 745204-1-745204-12, 2009.

[2] Durak M., Aslan H. M., "Optical characterization of the silicon photodiodes for the establishment of national radiometric standards" Optics Laser Technol. 36, 223-227, 2004.

[3] Robert D. Saunders and J. B. Shumaker, "Automated radiometric linearity tester", Applied Optics, Vol. 23, Issue 20, pp. 3504-3506 (1984).

[4] A. Thompson and H. M. Chen, "Beamcon III: a linearity measurement instrument for optical detectors," J. Res. Natl. Inst. Stand. Technol., 99, 751-755 1994.

[5] Tarde, R. and D. Heath, SCATS Accuracy Analysis and Budget Contributors, Ball Aerospace \& Technologies Corp., SER NO: CLARREO-001 R. Tarde, 05/18/2009.

[6] Cao, C., Wu, A., Xiong, X., and X. Wu, "Intercalibrating MetOP/AVHRR and AQUA/MODIS with Improved SNO Accuracy,” IGARSS 2007, Barcelona, IEEE, 2260-2263, 2007.

[7] Pollock, D.B., Murdock, T.L. and Datla, R.U., 2003, "Data uncertainty traced to SI units. Results reported in the international system of units," International Journal of Remote Sensing, 24, pp 225-235, 2003.

[8] Datla, R.U., et al., "Best practice guidelines for pre-launch characterization and calibration of instruments for remote sensing," Report to Global Space-based Inter-Calibration System (GSICS) NISTIR 7637, pp. 47, 2009.

[9] Datla, R.U., et al., "Uncertainty analysis of remote sensing optical sensor data: guiding principles to achieve metrological consistency," International Journal of Remote Sensing, 31, 867-880, 2010.

[10] Heath, D.F., and G. Georgiev," Characteristics of a New Type of Mie Scattering Volume Diffuser and its Use as a Spectral Albedo Calibration Albedo Calibration Standard for the Solar Reflective Wavelength Region," Proc. of SPIE Vol. 8153 Proceedings Earth Observing Systems XVI, 81530V-1, 2011.

[11] Bicout, D., C. Brosseau, A.S. Martinez, and J.M. Schmitt, "Depolarization of multiply scattered waves by spherical diffusers: Influence of the size parameter," Phys. Rev. E 49, pp 1767-1770, 1994.

[12] Nunes, E.H.M. and F.S. Lameiras, "The optical absorption of gamma irradiated and heat-treated natural quartz,” Materials Research, 8, pp. 305-308, 2005. 
[13] Balling, B, "A comparative study of the bidirectional reflectance distribution function of several surfaces as a mid-wave infrared diffuse reflectance standard," Master's Thesis, Air Force Institute of Technology, Graduate School of Engineering and Management (AFIT/GE/ENP/09-M01, (2009).

[14] Kitamura, R, Pilon, L., and M. Jonasz, "Optical constants of silica glass from extreme ultraviolet to far infrared at near room temperature," Appl. Opt., 46, 8118-8133, (2007).

[15] Rubens, H. and Nichols, E.F., "Certain optical and electro-magnetic properties of heat waves of great wavelength, I”, Phys. Rev., 5, 98-112, 1897.

APPENDIX

\begin{tabular}{|c|c|c|c|c|c|c|c|c|c|c|}
\hline \multicolumn{11}{|l|}{ Natural MSD } \\
\hline $\mathrm{AOI}=0 \mathrm{deg}$ & & & & & & & & & & \\
\hline $\begin{array}{l}\text { Wavelength } \\
\text { Scatter zenith }\end{array}$ & $250 \mathrm{~nm}$ & $280 \mathrm{~nm}$ & $300 \mathrm{~nm}$ & $350 \mathrm{~nm}$ & $400 \mathrm{~nm}$ & $500 \mathrm{~nm}$ & $700 \mathrm{~nm}$ & $900 \mathrm{~nm}$ & $1475 \mathrm{~nm}$ & $1575 \mathrm{~nm}$ \\
\hline 15 & 0.182 & 0.184 & 0.186 & 0.191 & 0.188 & 0.188 & 0.184 & 0.185 & 0.188 & 0.185 \\
\hline 30 & 0.181 & 0.180 & 0.185 & 0.189 & 0.188 & 0.186 & 0.182 & 0.183 & 0.184 & 0.181 \\
\hline 45 & 0.180 & 0.179 & 0.185 & 0.188 & 0.188 & 0.185 & 0.182 & 0.181 & 0.181 & 0.180 \\
\hline 60 & 0.177 & 0.177 & 0.185 & 0.186 & 0.187 & 0.184 & 0.182 & 0.180 & 0.182 & 0.181 \\
\hline 75 & 0.177 & 0.175 & 0.184 & 0.184 & 0.185 & 0.182 & 0.180 & 0.179 & 0.180 & 0.177 \\
\hline 135 & 0.114 & 0.117 & 0.122 & 0.128 & 0.130 & 0.132 & 0.134 & 0.143 & 0.152 & 0.152 \\
\hline 150 & 0.121 & 0.124 & 0.129 & 0.135 & 0.138 & 0.140 & 0.142 & 0.148 & 0.158 & 0.158 \\
\hline 165 & 0.125 & 0.128 & 0.133 & 0.138 & 0.141 & 0.144 & 0.145 & 0.151 & 0.163 & 0.161 \\
\hline 180 & 0.125 & 0.128 & 0.135 & 0.140 & 0.142 & 0.145 & 0.146 & 0.152 & 0.164 & 0.162 \\
\hline $\begin{array}{l}\text { Synthetic MS } \\
\mathrm{AOI}=0 \mathrm{deq}\end{array}$ & & & & & & & & & & \\
\hline Wavelength & $250 \mathrm{~nm}$ & 280nm & $300 \mathrm{~nm}$ & $350 \mathrm{~nm}$ & $400 \mathrm{~nm}$ & $500 \mathrm{~nm}$ & 700nm & $900 \mathrm{~nm}$ & 1475nm & $1575 \mathrm{~nm}$ \\
\hline Scatter zenith & & & & & & & & & & \\
\hline 15 & 0.220 & 0.220 & 0.221 & 0.222 & 0.222 & 0.221 & 0.216 & 0.215 & 0.214 & 0.212 \\
\hline 30 & 0.216 & 0.217 & 0.219 & 0.221 & 0.221 & 0.219 & 0.214 & 0.213 & 0.213 & 0.210 \\
\hline 45 & 0.213 & 0.216 & 0.218 & 0.220 & 0.220 & 0.218 & 0.213 & 0.211 & 0.209 & 0.208 \\
\hline 60 & 0.211 & 0.211 & 0.215 & 0.218 & 0.218 & 0.216 & 0.211 & 0.209 & 0.206 & 0.203 \\
\hline 75 & 0.204 & 0.210 & 0.215 & 0.216 & 0.216 & 0.213 & 0.207 & 0.206 & 0.203 & 0.201 \\
\hline 135 & 0.078 & 0.086 & 0.088 & 0.093 & 0.093 & 0.096 & 0.099 & 0.108 & 0.115 & 0.115 \\
\hline 150 & 0.085 & 0.091 & 0.095 & 0.099 & 0.099 & 0.103 & 0.106 & 0.112 & 0.120 & 0.120 \\
\hline 165 & 0.090 & 0.094 & 0.097 & 0.102 & 0.102 & 0.105 & 0.108 & 0.114 & 0.122 & 0.122 \\
\hline 180 & 0.096 & 0.099 & 0.104 & 0.107 & 0.107 & 0.111 & 0.112 & 0.117 & 0.127 & 0.126 \\
\hline
\end{tabular}

\begin{tabular}{|c|c|c|c|c|c|c|c|c|c|c|}
\hline \multicolumn{11}{|l|}{ QVD } \\
\hline \multicolumn{11}{|l|}{$\mathrm{AOI}=0 \mathrm{deg}$} \\
\hline Wavelength & $250 \mathrm{~nm}$ & $280 \mathrm{~nm}$ & $300 \mathrm{~nm}$ & $350 \mathrm{~nm}$ & $400 \mathrm{~nm}$ & $500 \mathrm{~nm}$ & $700 \mathrm{~nm}$ & 900nm & $1475 \mathrm{~nm}$ & $1575 \mathrm{~nm}$ \\
\hline \multicolumn{11}{|c|}{ Scatter zenith } \\
\hline 15 & 0.170 & 0.170 & 0.175 & 0.177 & 0.179 & 0.177 & 0.170 & 0.177 & 0.191 & 0.196 \\
\hline 30 & 0.161 & 0.162 & 0.166 & 0.168 & 0.171 & 0.168 & 0.161 & 0.166 & 0.183 & 0.187 \\
\hline 45 & 0.153 & 0.152 & 0.157 & 0.161 & 0.162 & 0.159 & 0.152 & 0.156 & 0.171 & 0.173 \\
\hline 60 & 0.148 & 0.147 & 0.154 & 0.157 & 0.157 & 0.155 & 0.147 & 0.152 & 0.167 & 0.171 \\
\hline 75 & 0.144 & 0.145 & 0.155 & 0.159 & 0.160 & 0.157 & 0.150 & 0.153 & 0.174 & 0.176 \\
\hline
\end{tabular}

